\title{
Robot Path Planning Based on Genetic and Chaotic Optimization Algorithm
}

\author{
Gan Xusheng, ${ }^{1, a}$, Gao Wenming ${ }^{2, b}$, Han Jun ${ }^{2, c}$ \\ ${ }^{1}$ XiJing College, Xi’an, Shaanxi, 710123, China; \\ 2.Air Traffic Control and Navigation College, Air Force Engineering University, Xi’an, Shaanxi, 710051, \\ China; \\ aganxusheng123@163.com; '3912yhk@163.com; cxbs421@yahoo.com.cn
}

Keywords: Robot, Path Planning, Genetic Algorithm, Chaotic Optimization Algorithm.

\begin{abstract}
For robot path planning for avoiding the obstacle, a path planning method based on modified Genetic Algorithm (GA) is proposed. In the algorithm, GA is first used to roughly search the path for robot as a whole, and then chaotic optimization algorithm is adopted to perform the refined search on the basis of the result obtained by GA, which can make remarkable progress in accuracy and speed. The simulation result shows that the proposed algorithm is more reasonable and effective for robot path planning.
\end{abstract}

\section{Introduction}

Intelligent robot path planning technology determines the level of autonomy. The technology has been paid more and more domestic and foreign scholars and engineering researcher's attention. It has been widely used in the civilian areas such as social security, geodetic mapping, marine testing, submarine survey, rescue and so on, as well as the people's daily life. Especially in recent years, with popularity of online shopping mode, freight volume is also growing. For this, some businesses put forward 'send express by robot' concept, which are inseparable from the research on robot path planning. To realize these concept, the robot is required to avoid obstacles in real-time manner by reasonable optimal path planning for ensuring own safety operation [1].

At present, there are many researches on robot path planning at home and abroad, especially the use of some intelligent optimization algorithm has achieved good results. Particle Swarm Optimization (PSO) algorithm is a method finding the optimal solution through continuous search and adjustment, which is widely used to solve the optimal problem and is suitable for solving the robot path planning problem. In recent years, there are many problems in robot path planning by PSO algorithm. For example, Li Meng proposed a method that integrates threat-enlightened mechanism into PSO algorithm in order to improve the search ability of particle [2]. Zhang Renpeng established the particle concentration mechanism so as to analyze the concentration of particle swarm falling into the local optimum for improving the planning efficiency [3]. Liu Ke introduced the complex network theory to modify the PSO algorithm by adjusting the inertia coefficient automatically for improving convergence ability [4]. Liu Huizhuo selected tabu search method to solve the premature and local convergence problems of PSO algorithm [5]. These have achieved good experimental results. After a lot of reading literature, the space division and a modified genetic algorithm are used to the study the robot path planning. Simulation results show that the proposed method is suitable for solving robot path planning.

\section{Robot Path Planning Principle}

The path planning is to plan the path from the initial position to the target location without collision according to certain optimization strategy for the given initial position and the target position in a certain obstacle environment. Its application in this paper is that the express robot 
autonomously finds one optimal line to send the goods from the warehouse to the customer's hands. The difficult problem is how to create one path with the shortest distance and smooth line for the robot while avoiding obstacles.

In the paper, the path planning problem of express robot is studied under the static environment in three-dimensional space. The path planning under the static environment is to find an optimal path from the initial point to the target point according to some optimization criteria. A robot sends the goods from the initial point to the target point, in the middle path, there are a number of obstacles. The obstacles, the initial point A and the target point B are shown using 3D topographic map. Through the environment modeling, the intelligent search algorithm is selected to search the path.

Firstly, through fast natural neighbor interpolation method, several obstacle objects can be constructed to form a 3D topographic view. Then, the abstract modeling on 3D environment space can be carried out from 3D terrain data to create the environment space of path planning, the coordinate axes A-XYZ, the cube ABCD-EFGH, as shown in Fig. 1.

The cube is firstly rasterized, namely the edge $A E$ is divided into $n$ equal parts to get $n+1$ planes $\prod i(i=0,1,2, \ldots, n)$. Then for any plane $\prod i$, the edge $A^{\prime} B^{\prime}$ is divided into $l$ equal parts. In this way, the plane $\prod i$ is divided into $m \times l$ lattice cells, which endpoints act as the path nodes for the robot, and the set of these endpoints can be expressed as $s^{*}$, as shown in Fig. 2.

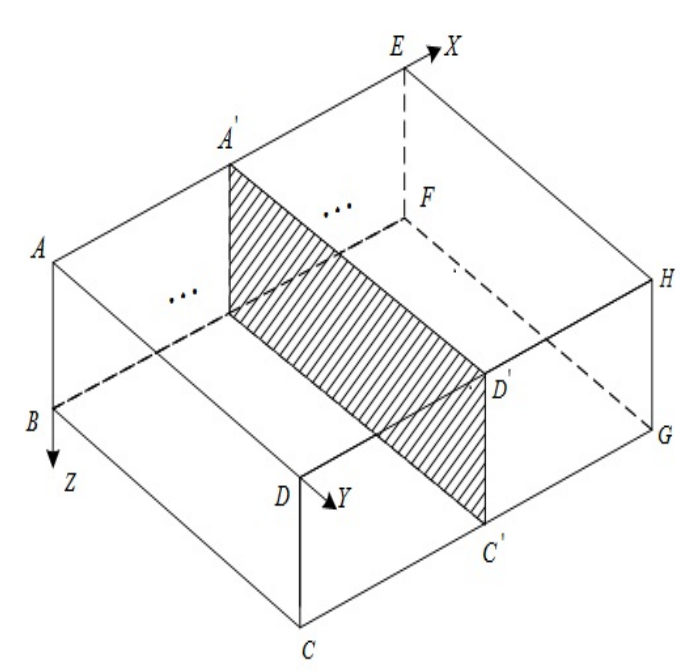

Fig. 1. 3D coordinate cube view

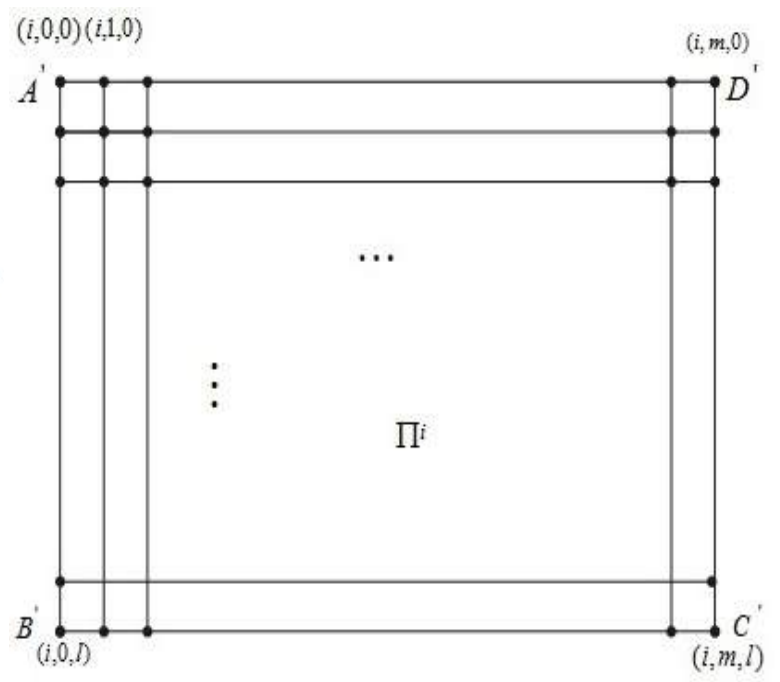

Fig. 2. Cube arbitrary face $\prod i$ grid division

A point $p$ in the set corresponds to 2 coordinates: ordinal coordinate $p^{1}(i, j, k)$ and position coordinate $p^{2}\left(x_{i}, y_{j}, z_{k}\right)(i=\{0,1,2, \cdots, n\}, j=\{0,1,2, \cdots, m\}, k=\{0,1,2 \cdots, l\})$, where $i, j, k$ denote the division number of the points $p$ along $A E, A D$ and $A B$. The ordinal coordinate of the vertex $A$ of path planning environment space is $p^{1}(0,0,0)$, and the position coordinate is $p^{2}(0,0,0)$. Assume that $|A E|=\Delta \phi, \quad|A D|=\Delta \lambda,|A B|=h$. The position coordinates of the point $p$ can be expressed as

$$
\left\{\begin{array}{l}
x_{i}=(i \cdot \Delta \phi) / n \\
x_{j}=(j \cdot \Delta \lambda) / m \\
x_{k}=(k \cdot h) / l
\end{array}\right.
$$

For a point in the point set $s^{*}$ obtained by discretizating the path planning space, a 2-tuple $<p$, state $>$ is used to store the information, where $p$ represents the position of the point, state represents the state (freedom or obstacle) of the change point. If the position of the point $p$ is the obstacle area, let state $=0$, it is the obstacle point; otherwise, let state $=1$, it is the freedom point. When judge whether a point $p$ in the point set $s^{*}$ is the obstacle point or freedom point, at first, the depth of the corresponding latitude and longitude position of the point in the three-dimensional terrain data are 
calculated. If the coordinate value $z_{k}$ is less than the depth, then the point $p$ is the freedom point, otherwise the point $p$ is the obstacle point.

The distance between the points on two adjacent planes $\prod i$ and $\prod i+1$ is defined as $s\left(p_{i}, p_{i+1}\right)$, namely the distance between two adjacent nodes of the robot. The calculation formula is as follows

$$
s\left(p_{i}, p_{i+1}\right)=\sqrt{\left(x_{i+1}-x_{i}\right)^{2}+\left(y_{i+1}-y_{i}\right)^{2}+\left(z_{i+1}-z_{i}\right)^{2}}
$$

Finding the optimal path of the robot is to calculate the shortest distance between the point sequence, and the formula is as follows:

$$
d_{A B}=\min \sum_{i=0}^{n+1} \sqrt{\left(x_{i+1}-x_{i}\right)^{2}+\left(y_{i+1}-y_{i}\right)^{2}+\left(z_{i+1}-z_{i}\right)^{2}}
$$

where $\left(x_{i}, y_{i}, z_{i}\right),\left(x_{i+1}, y_{i+1}, z_{i+1}\right)$ are the coordinates of the node, $n+1$ is the number of nodes.

On the basis of rasterization process, a modified Genetic Algorithm (GA) based on chaos theory is selected to optimize the robot path.

\section{Chaos Genetic Algorithm}

\subsection{Genetic Algorithm}

GA is a heuristic search algorithm on the basis of the theory of evolutionism and genetics. In the algorithm, firstly the search space is mapped into the genetic space, and the possible solution is encoded to form the so-called chromosome and population, and then according to the 'survival of the fittest' principle the chromosomes suitable for the environment are selected from the population to perform the genetic operations such as reproduction, crossover and mutation for next generation population. The above process is repeated until the optimal solution.

In GA algorithm, the definite encoding technique and reproduction mechanisms are used to express a very complex phenomenon, solving some difficult optimization problem. It is a stochastic optimization method with the advantages of relaxation of initial values, strong global search ability, robustness suitable for a wide problem domain, stable solution, connotative parallelism without differentiability requirement. However, for solving the large complex systems, especially the optimization problem of nonlinear system, SGA has still some shortcomings such as unable guarantee for the global convergence, loss of the best chromosome in the population, premature convergence of evolution process, etc.

\subsection{Chaotic Optimization Algorithm}

Basic idea of Chaotic Optimization Algorithm (COA) is that, at first the chaos variables in the chaotic space are mapped into the solution space, then the good characteristics of the chaos variable are used to search the optimal solution. In general, the optimization process may be divided into two stages: first each point passed is traversed in turn within values range of variable, accepting the best point as the optimal solution; then a chaos small-perturbation is added to the current optimal solution in order to refinedly search the optimal value. COA is sensitive to initial value with a fast search rate, and is easy to get out of local minimum. But the optimal effect is not satisfactory for a large search space.

Logistic mapping is easy to use with less computation, and widely used. Its time series can be defined as

$$
x_{k+1}=\mu x_{k}\left(1-x_{k}\right)
$$

where $x \in(0,1)$; the bifurcation parameter $\mu=[1,4]$; when $\mu$ is the value in [3.56994, 4], the system is in the chaotic region where the trajectory of the mapping equation shows the chaos characteristic. For the chaos mapping $x_{k+1}=f\left(x_{k}\right)$, the probability density function $\rho(x)$ can be obtained by the Perron-Froenious equation 


$$
\rho(x)= \begin{cases}\frac{1}{\pi \sqrt{x(1-x)}} & x \in(0,1) \\ 0 & \text { other }\end{cases}
$$

The mean of $x$ is

$$
\bar{x}=\int_{0}^{1} x \rho(x) d x=\frac{1}{2}
$$

\subsection{Combination of GA and COA}

According to above analysis, GA is not sensitive to initial values with the global search capability, but it cannot ensure to converge to the global optimum, and easily falls into local optimum. COA is sensitive to initial values, and because of its ergodicity can avoid falling into local optimum, but it usually requires large numbers of iterations to get a better solution.

Based on this, it should be considered that, GA is integrated with COA, constructing a hybrid algorithm: CGA, which is expected to provide a stronger optimal capability [6][7]. In CGA, GA is mainly used to perform the global search, and COA is adopted to conduct the local search on the basis of the results obtained by GA. Thus, theoretically, CGA can improve the global search efficiency and avoid falling into local optimum [8]. The steps of CGA are as follows:

1. Conduct the binary or decimal code for the parameters to be optimized, constituting the initial population, and calculate the fitness value of each individual.

2. Select the individual into the next generation by the probability $p_{i}=f_{i} / \sum_{i} f_{i}$.

3. Perform the crossover operation for the individuals selected by the probability $p_{c}$.

4. Perform the mutation operation for the individuals selected by the probability $p_{m}$.

5. Decode, and calculate the fitness value of each individual.

6. Conduct the chaos optimization for the individuals that have the best fitness value.

7. If the fitness value of the new individual is better than that of the original individual, the original individual is replace, otherwise, the original individual doesn't need to be changed.

8. Repeat from step 2 to step 7 until the terminal condition is satisfied.

\section{Experiment Simulation}

In order to validate the effectiveness of path planning method proposed in the paper, Matlab programming language is used to simulate the experiment. The airspace flown by robot can be selected as $120 \mathrm{~km} \times 120 \mathrm{~km} \times 80 \mathrm{~km}$. The starting point A of robot is $(5,100,8)$, the target point $B$ is $(95,10,10)$. In flight area, there are 6 peaks as terrain threat, and 2 dangerous areas, as shown in Fig. 3. The parameter settings: The population size is taken as 30 . The maximum evolution generation is 200, $p_{c}=0.85, p_{m}=0.1, \mu=4$. 


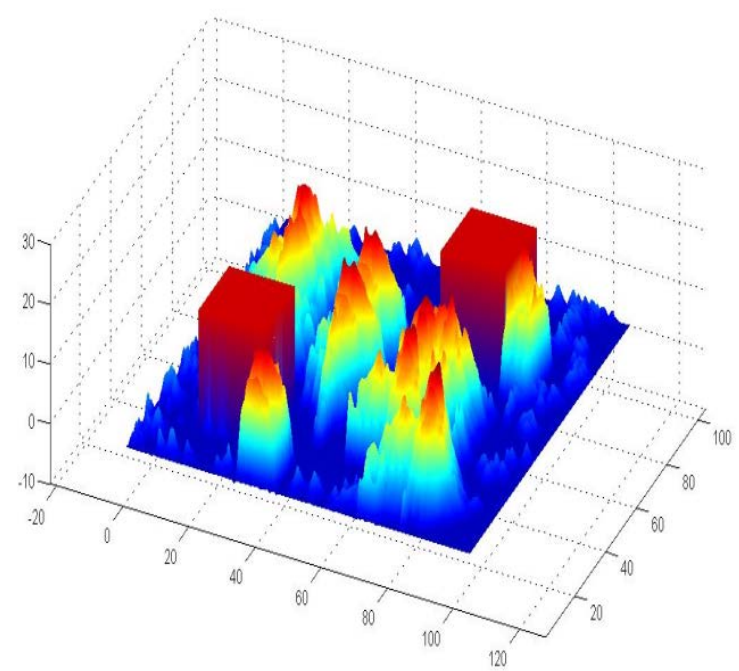

Fig. 3. Airspace operational environment

The comparison and analysis for robot path planning using traditional GA algorithm, modified GA algorithm, CGA algorithm based on space division are given in Fig. 4, Fig. 5 and Fig. 6.

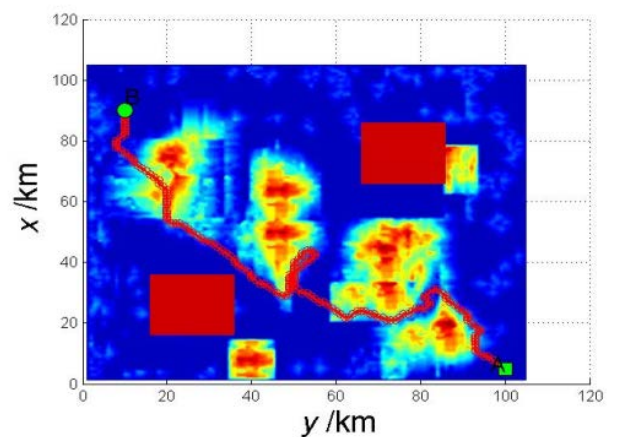

(a) $90^{\circ}$ vertical view

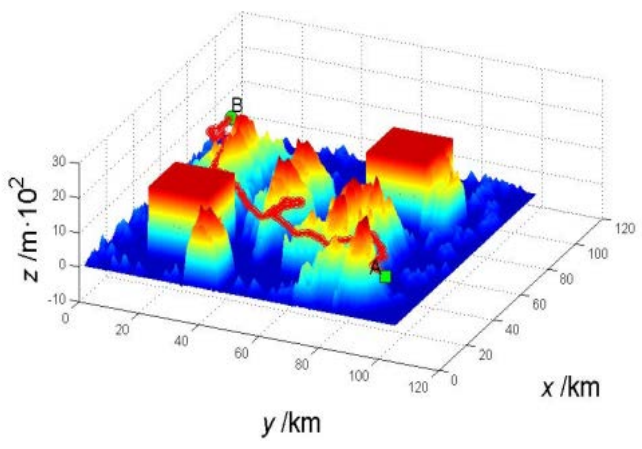

(b) $45^{\circ}$ vertical view

Fig. 4. Robot path planning using traditional GA algorithm

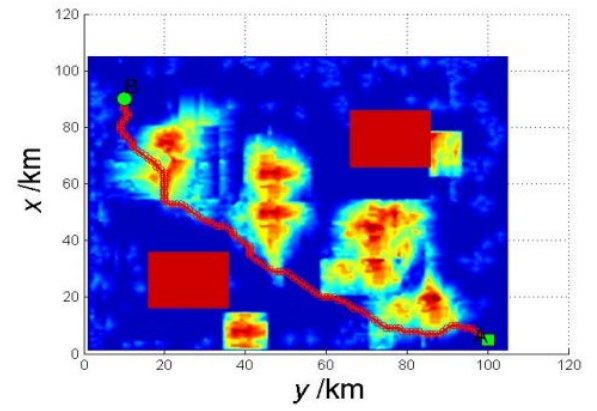

(a) $90^{\circ}$ vertical view

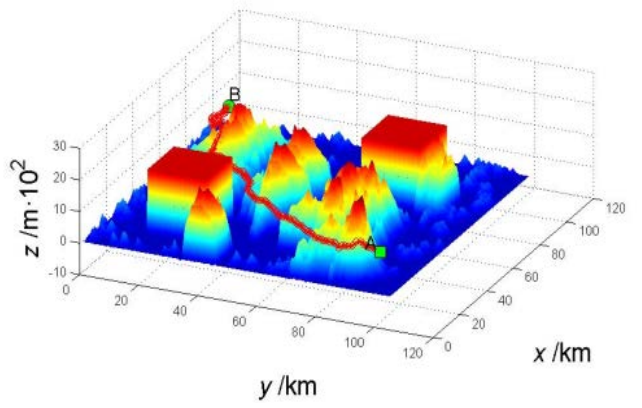

(b) $45^{\circ}$ vertical view

Fig. 5. Robot path planning using modified GA algorithm 


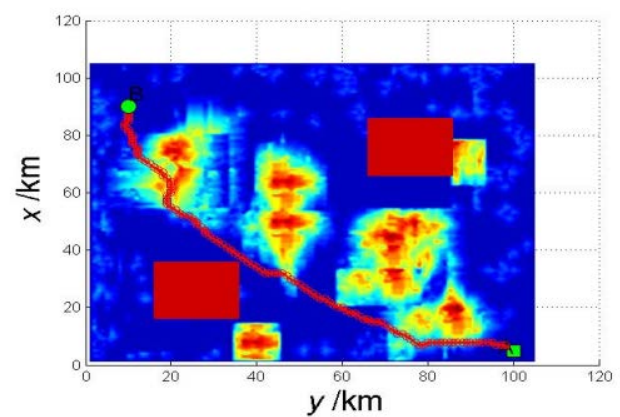

(a) $90^{\circ}$ vertical view

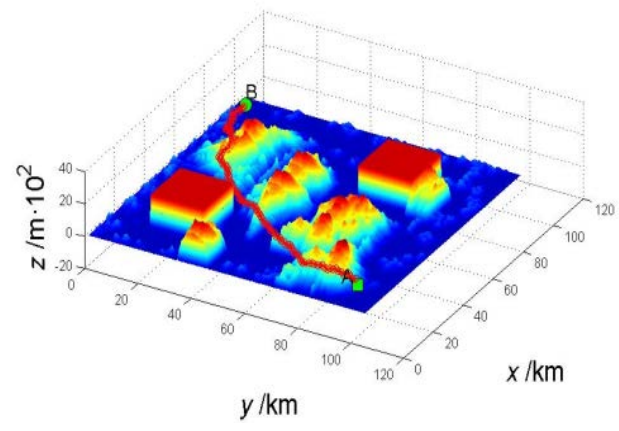

(b) $45^{\circ}$ vertical view

Fig. 6. Robot path planning using CGA algorithm based on space division

It can be seen from Fig. 4 6 that, there is a certain amount of redundancy in optimal path planning of the robot using traditional GA algorithm and modified GA algorithm, which shows that the local optimum is found in the optimization process. With the increase of the flight distance it increases the contact time with the obstacle, which is detrimental to the safety of the robot. However, under same parameter setting, the effect of path planning using CGA algorithm based on space division is more accurate and shorter than that of traditional GA algorithm and modified GA algorithm, which can guarantee the safety. This reduces the maneuver of the robot and the flight of the robot is more stable and the operation is safe.

\section{Conclusion}

In order to solve the path planning problem of robot for avoiding the obstacle, a modified genetic algorithm (GA) integrating with chaos theory on basis of space division is proposed. Through environment modeling, the airspace separation, the method effectively improves the quality of path planning and efficiency.

\section{References}

[1] L. Q. Liu, G. Li, Y. X. Zhao, "Mission planning for UAV," Published by Beijing Science Press, 2014.

[2] M. Li, D. B. Wang, T. T. Bai, et al, "Route planning based on particle swarm optimization with threat heuristic,” Electronics Optics \& Control, vol. 18, No. 12, 2011, pp. 1-4.

[3] R. P. Zhang, J. X. Yang, J. H. Pan, et al, "Three-dimensional route planning of UAV based on improved particle swarm algorithm,” Computer Simulation, vol. 31, No. 3, 2014, pp. 65-69.

[4] K. Liu, J. Q. Zhou, X. H. Guo, "Path planning research for unmanned air vehicle based on improved particle swarm algorithm,” Journal of North University of China, vol. 34, No. 4, 2013, pp. 441-446.

[5] H. Z. Liu, J. X. Yang, T. R. Wang, "Route planning in the three-dimensional space using improved adaptive PSO,” Fire Control \& Command Control, vol. 38, No. 11, 2013, pp. 141-143.

[6] B. Li, W. S. Jiang, "Chaos optimization method and Its application," Control Theory and Application, vol. 14, No. 4, 1997, pp. 613- 615.

[7] J. F. Yao, C. Mei, X. Q. Peng, etc, “A new optimization approach-chaos genetic algorithm,” System Engineering, vol. 19, No. 1, 2001, pp. 70-74.

[8] S. T. Yi, J. T. Tang, G. L. Nie, "Improved chaos genetic algorithms," Changsha Aeronautical Vocational and Technical College Journal, vol. 4, No. 3, 2004, pp. 35-37. 